\title{
Effects of Statin Use for Primary Prevention among Adults Aged 75 Years and Older in the National Health Insurance Service Senior Cohort (2002- 2015)
}

\author{
Sunyoung Kim ${ }^{1}$, Hangseok $\mathrm{Choi}^{2}$, Chang Won Won ${ }^{3}$ \\ ${ }^{1}$ Department of Family Medicine, Kyung Hee University Medical Center, Seoul, Korea \\ ${ }^{2}$ College of Pharmacy, Chung-Ang University, Seoul, Korea \\ ${ }^{3}$ Elderly Frailty Research Center, Department of Family Medicine, College of Medicine, Kyung Hee University, Seoul, Korea
}

\author{
Corresponding Author: \\ Chang Won Won. MD, PhD \\ Elderly Frailty Research Center, \\ Department of Family Medicine, \\ College of Medicine, Kyung Hee \\ University, 23 Kyungheedae-ro, \\ Dongdaemun-gu, Seoul 02447, Korea \\ E-mail: chunwon@khmc.or.kr \\ ORCID: \\ https://orcid.org/0000-0002-6429-4461
}

Received: May 7, 2020

Revised: June 16, 2020

Accepted: June 17, 2020
Background: This study aimed to identify adverse events and mortality in adults aged 75 years and older who were initially prescribed statins for primary prevention. Methods: This retrospective study analyzed the data from the National Health Insurance Corporation-Senior Cohort from 2002 to 2015. An exact block matched model was constructed from statin user and statin non-user groups. Results: The study sample comprised 1,370 older adults (mean age, 78 years), with 685 statin non-users matched to 685 new statin users. Compared to non-users, the adjusted hazard ratios (HRs) of new statin users were $0.83(p=0.04)$ for all-cause mortality, 1.24 $(p=0.003)$ for major adverse cardiovascular events, and $1.18(p=0.06)$ for new-onset diabetes mellitus. In a sub-analysis of statin use duration, longer statin use ( $>5$ years) was associated with a significantly lower risk of all-cause mortality ( $H R=0.76, p=0.01)$ but not with major adverse cardiovascular events ( $H R=0.88, p=0.36$ ) or new-onset diabetes mellitus ( $H R=0.95, p=0.78$ ) after adjusting for age, sex, body mass index, diabetes mellitus, hypertension, aspirin use, and antiplatelet use. Conclusion: Our findings suggested that statins started for primary prevention in older adults aged 75 years and older had an advantageous effect on all-cause mortality only if used for at least 5 years.

Key Words: Aged, Cardiovascular disease, Hydroxymethylglutaryl-CoA reductase inhibitors, Mortality, Primary prevention

\section{INTRODUCTION}

Among countries with an aging global population, South Korea is the country with the fastest-aging population; with more than $14 \%$ of its population aged 65 years and older, South Korea officially became an aged society in 2017. ${ }^{1)}$ Cardiovascular disease is the second leading cause of death among Koreans, and ischemic heart disease accounts for the majority of deaths.

High low-density lipoprotein cholesterol (LDL-C) level is a well-documented risk factor for cardiovascular disease because it is associated with progression from early-stage fatty streaks to advanced-stage, lipid-rich plaques. ${ }^{3)}$ Hydroxymethylglutaryl-coen- zyme A reductase inhibitors, are one of the best-established means for preventing and treating atherosclerotic cardiovascular disease. ${ }^{4)}$ However, there is limited evidence to recommend statins for the primary prevention of cardiovascular disease in adults aged 75 years and over. ${ }^{5,6)}$

Since the American College of Cardiology/American Heart Association (ACC/AHA) guidelines published in $2013,{ }^{5)}$ five guidelines for statin use have been released: ${ }^{7)}$ the UK National Institute for Health and Care Excellence (NICE-UK) in 2014, ${ }^{8}$ the Canadian Cardiovascular Society (CCS), ${ }^{9}$ the US Preventive Services Task Force, ${ }^{6}$ the European Society of Cardiology/European Atherosclerosis Society $(\mathrm{ESC} / \mathrm{EAS})^{10)}$ in 2016 , and ACC/AHA in 
2018. ${ }^{11)}$ However, of these guidelines, only the NICE-UK strongly recommends statins for primary prevention up to age 84 ; the ESC/EAS and the ACC/AHA recommend treatment to age 65 and 75 , respectively. The Korean guideline for dyslipidemia in 2018 indicated that there is insufficient evidence regarding the effectiveness of statins for primary prevention of cardiovascular disease in older adult patients over 75 years of age without cardiovascular disease and diabetes and recommended to consider statin use for primary prevention only in case of diabetes. ${ }^{12)}$

Previous studies reported increased risks of diabetes mellitus $(\mathrm{DM})^{13)}$ and cognitive dysfunction due to statin use, ${ }^{14)}$ with concerns regarding statin prescriptions for older adults. However, few studies have evaluated the efficacy and safety of statins in people aged 75 years or older.

Furthermore, most studies were conducted in Western countries; thus, there is insufficient clinical evidence regarding the primary preventive effect or tolerability of statins in older Asian populations.

Therefore, we evaluated the efficacy, mortality, and adverse outcomes of statins in people aged over 75 years by analyzing National Health Insurance data.

\section{MATERIALS AND METHODS}

\section{Data Background}

The dataset was provided by the Korean National Health Insurance Service (NHIS), which was founded in 2000 as a single-insurer system. The NHIS has converted all medical records into the National Health Information Database containing personal information, demographic data, and medical treatment data for Korean citizens categorized as insured employees, insured self-employed individuals, or medical-aid beneficiaries. Within this dataset is the NHIS-Senior Cohort (NHIS-SC), which is a population-based cohort comprising 558,147 people who account for approximately $10 \%$ of the total $5,500,000$ patients aged $\geq 60$ years in 2002 . The NHIS-SC database contains information on insurance membership and income, medical use history, medical checkups, and longterm care and provides data from medical health examinations that all older adult beneficiaries receive every 2 years, including blood pressure measurement, health behavior status (smoking, alcohol), and past medical history. ${ }^{15,16)}$

\section{Study Population}

In this study, we included people aged 75 years and older who were enrolled in the NHIS-SC database between January 1, 2004, and December 31, $2005(n=159,015)$ and who received a health examination provided by the NHIS within 2 years of their first pre- scription day $(n=25,350)$. We included people with total cholesterol level of $>200 \mathrm{mg} / \mathrm{dL}$ on their health screening blood test. The exclusion criteria were: (1) having been prescribed statins from 2002-2003 or (2) having any of the following major or minor diagnostic codes on their medical records within the 2 years before enrollment -I20-25 (ischemic heart disease), I60-69 (cerebrovascular disease), and I73 (other peripheral vascular diseases). For comparison, the baseline cohort included patients with hypercholesterolemia (with claim codes or a total cholesterol level $>200$ $\mathrm{mg} / \mathrm{dL}$ ) in the primary prevention cohort who were eligible for statin therapy but had no statin prescription history $(n=7,782)$. The medication possession ratio (MPR) was calculated by dividing the sum of prescription days by the total prescription period (between the first date of prescription and the final follow-up day during the index period; January 1, 2004 to December 31, 2005) using medication history extracted from each patient's medical record. Subjects with a statin MPR above $20 \%$ were assigned to the statin-user group $(n=767)$, while those who had never been exposed to statins were assigned to the statin non-user group $(n=15,637) .{ }^{17,18)}$ Because statin use was not randomly assigned, the effects of treatment-selection bias and potential confounding factors were mitigated by applying an exact block-matching approach. Exact block matching was determined using four blocks of sex, age (age groups within 2 years), total cholesterol (three categories; < 240, 240-300, and > $300 \mathrm{mg} / \mathrm{dL}$ ), and incidence year, with each statin user matched to one control subject. The analyses finally included 685 pairs $(n=1,370)$ (Fig. 1).

\section{Covariate Confounders}

We considered the risk factors for adverse events, including underlying medication history, socio-demographic characteristics (age, sex, income), body mass index (BMI), and lifestyle (smoking, alcohol consumption), as potential confounders.

\section{Outcomes}

The primary outcome variables included the rates of all-cause death and major adverse cardiovascular events (MACE), which was defined as the composite of myocardial infarction (MI; I2025), stroke (I60-69), and coronary revascularization (I46; percutaneous transluminal coronary angioplasty or coronary artery bypass grafting) from NHIS-SC records dated within the study period. The secondary outcomes were hospitalization for any cause (acute MI, ischemic cerebrovascular disease, chronic obstructive pulmonary disease, and malignant neoplasm), cancer, new-onset diabetes mellitus (NODM), liver toxicity, and myopathy. ${ }^{19)}$ NODM was recorded when antidiabetic drugs were first prescribed during follow-up and when diagnostic codes were claimed for the first time. 


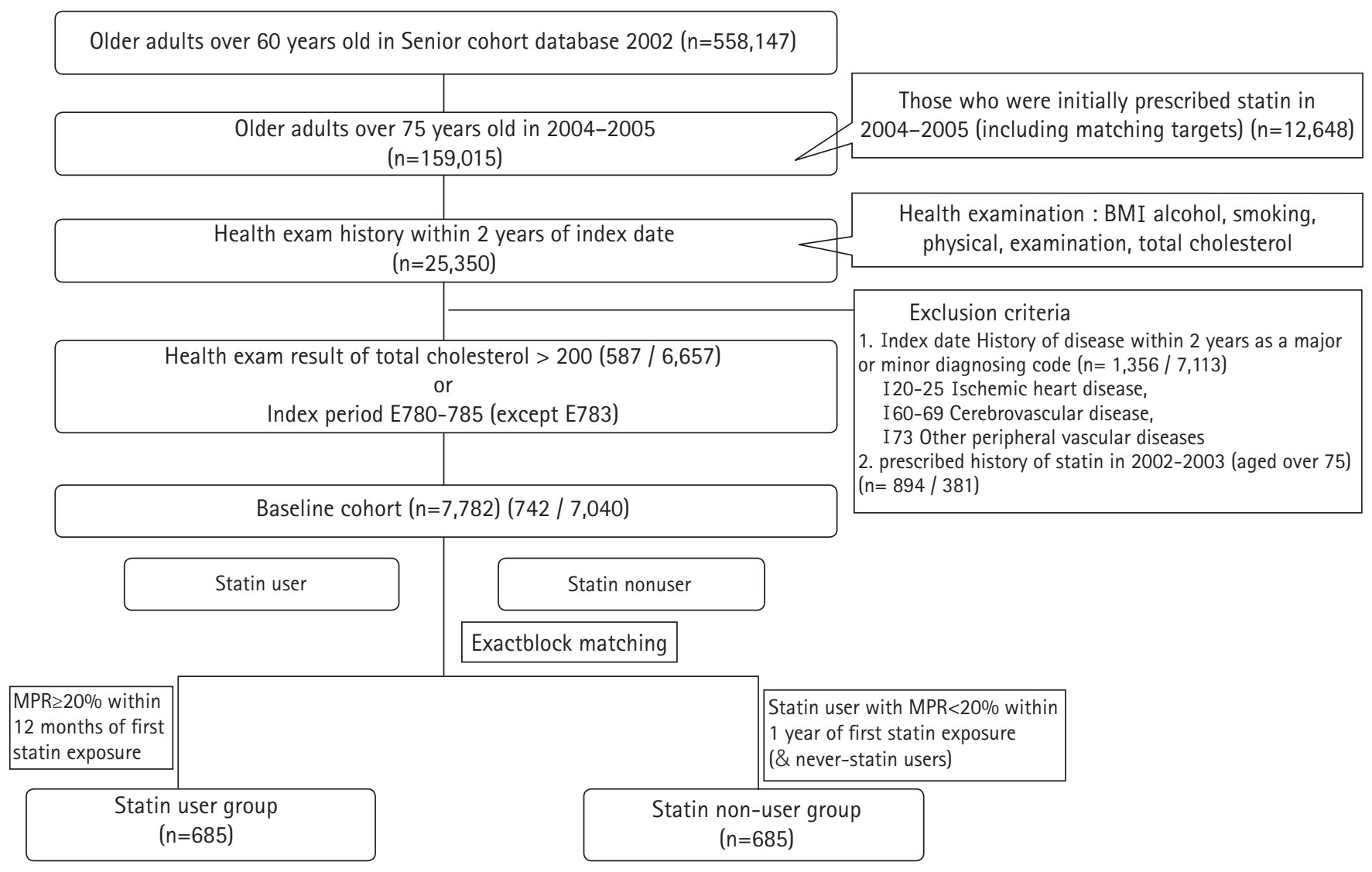

Fig. 1. Flow chart of study subjects. BMI, body mass index; MPR, medication possession ratio.

The DM diagnostic codes were taken from the 10th revision of the International Statistical Classification of Diseases as follows: E11 (non-insulin-dependent DM), E12 (malnutrition-related DM), E13 (other specified DM), or E14 (unspecified DM). The classes of antidiabetic drugs recognized for NODM diagnosis were sulfonylureas, biguanides, alpha-glucosidase inhibitors, thiazolidinediones, meglitinides, and insulin. ${ }^{20)}$ Incretin-based therapies (i.e., glucagon-like peptide- 1 receptor agonists and dipeptidyl peptidase-4 inhibitors) had not yet been introduced during the study period. Liver toxicity (K71, toxic liver disease) and myopathy (G71, drug-induced myopathy; M62.8, rhabdomyolysis) were considered attributable to statins if they occurred within 12 months of initiation. ${ }^{21)}$

\section{Ethics Statement}

This study was approved by the Kyung Hee University Hospital Research Ethics Committee (No. KMC IRB 1601-09).

\section{Statistical Analyses}

We defined overall survival, one of the primary endpoints of this study, as the time from the enrollment date to the date of death from any cause. The baseline characteristics of the two groups are expressed as means and standard deviations for continuous variables and as percentages for categorical variables. We used Wilcoxon two-sample and Fisher exact tests for continuous and categorical variables, respectively. We performed log-rank tests and Cox proportional hazard regression to examine the differences between the statin user and non-user groups. Finally, we estimated the hazard ratios (HRs) adjusted for age, sex, BMI, DM, hypertension (HTN), aspirin, and antiplatelet use. All statistical analyses were conducted using R version 3.4.4 (R Foundation for Statistical Computing, Vienna, Austria) and SAS version 9.3 (SAS Institute Inc., Cary, NC, USA) to explore and modify the large datasets.

\section{RESULTS}

\section{General Characteristics}

The average age of the 1,370 people who met the inclusion criteria was 78 years. The mean follow-up period was 8.7 years (statin users 8.7 years vs. statin non-users 8.6 years). We analyzed 685 pairs by propensity matching for sex, cholesterol level, and residential area. Statin users were more likely to have HTN and DM and to be 
Table 1. Baseline characteristics

\begin{tabular}{|c|c|c|c|}
\hline \multirow{2}{*}{ Variable } & \multicolumn{3}{|c|}{ Exact matched cohort } \\
\hline & Statin users $(n=685)$ & Statin non-users $(n=685)$ & $\mathrm{p}$-value \\
\hline Age (y) & $78.0 \pm 3.0$ & $78.1 \pm 3.2$ & 0.900 \\
\hline Sex, women & $450(65.7)$ & $450(65.7)$ & 1.000 \\
\hline HTN & $548(80.0)$ & $267(39.0)$ & $<0.001$ \\
\hline Diabetes mellitus & $241(35.2)$ & $90(13.1)$ & $<0.001$ \\
\hline Aspirin use & $130(19.0)$ & $31(4.5)$ & $<0.001$ \\
\hline Antiplatelet use & $185(27.0)$ & $39(5.7)$ & $<0.001$ \\
\hline HTN medication use & $460(67.2)$ & $200(29.2)$ & $<0.001$ \\
\hline Alcohol drinker & $51(7.4)$ & $44(6.4)$ & 0.520 \\
\hline Smoker & $86(12.6)$ & $101(14.7)$ & 0.270 \\
\hline $\mathrm{BMI}\left(\mathrm{kg} / \mathrm{m}^{2}\right)$ & $23.9 \pm 3.3$ & $23.2 \pm 3.3$ & $<0.001$ \\
\hline Physically active & $130(19.0)$ & $108(15.8)$ & 0.130 \\
\hline Total cholesterol (mg/dL) & $233.2 \pm 44.0$ & $237.7 \pm 39.9$ & 0.180 \\
\hline
\end{tabular}

Values are presented as mean \pm standard deviation or number (\%).

HTN, hypertension; BMI, body mass index.

taking aspirin or other antiplatelet. We observed no significant differences in age distribution, alcohol, smoking, or physical activity between the statin user and non-user groups (Table 1).

\section{Outcomes according to Statin Use}

During follow-up, significantly higher rates of MACE (HR $=1.51 ; \mathrm{p}<0.001)$, hospitalization $(\mathrm{HR}=1.21$, $\mathrm{p}<0.001)$, and $\operatorname{NODM}(\mathrm{HR}=1.28, \mathrm{p}=0.002)$ were observed in the statin user group than in the non-user group. In addition, significantly higher rates of $\mathrm{MI}(\mathrm{HR}=1.25, \mathrm{p}<0.001)$ and stroke $(\mathrm{HR}=1.21$, $\mathrm{p}=0.026)$ and a non-significantly higher rate of coronary revascularization $(\mathrm{HR}=1.25, \mathrm{p}=0.750)$ were observed in the statin user group than in the non-user group (Supplementary Table S1). After adjusting for age, sex, BMI, DM (except for NODM), aspirin use, and antiplatelet use, the HRs were $0.83(\mathrm{p}=0.040)$ for all-cause mortality, $1.24(p=0.030)$ for MACE, and 1.18 $(p=0.060)$ for NODM. The risks of hospitalization, cancer, myopathy, and hepatitis did not differ significantly according to statin use (Table 2).

\section{Subgroup Analysis for Stain Use Duration}

Our sub-analysis of duration showed a significant relationship between longer statin use ( $>5$ years) and all-cause mortality $(\mathrm{HR}=0.76, \mathrm{p}=0.001)$ after adjusting for age, sex, BMI, DM, HTN, aspirin use, and antiplatelet use. The MACE risk was elevated among patients who used statins for $<3$ years $(\mathrm{HR}=1.5$, $\mathrm{p}<0.001$ ), but MACE risk was lower among patients with statin use duration $>5$ years $(\mathrm{HR}=0.88, \mathrm{p}=0.360)$. Similarly, NODM risk was higher for $<3$-year statin use duration $(H R=1.10$, $\mathrm{p}=0.190)$ but lower for $>5$ years duration $(\mathrm{HR}=0.95$, $\mathrm{p}=0.780$ ). These results suggest that patients receiving statins for longer periods were less likely to experience adverse events, including all-cause mortality, MACE, and NODM (Table 3).

\section{DISCUSSION}

The most important finding in this study was that statin use for primary prevention in patients aged over 75 years was associated with a lower risk of all-cause mortality and higher risks of MACE and NODM. Longer statin use ( $>5$ years) resulted in a significantly lower risk of all-cause mortality and did not result in a significantly higher risk for NODM, hospitalization, or cancer compared to those for non-use. Finally, while the risk of MACE in long-term statin use was not statistically significant, it tended to decrease.

Advanced age is recognized as a definite and strong risk factor for cardiovascular disease. ${ }^{22)}$ The clinical benefit of statin use for secondary prevention, even in older adult patients, has been robustly demonstrated. ${ }^{23)}$ However, direct evidence of a benefit for primary prevention in patients aged 75 years and over is controversial. $^{24)}$ Although the reason for this controversy is not clear, differences in sex, study design, participants, age range, and ethnic characteristics between studies may contribute to these differences. ${ }^{25)}$ The participants included in previous trials of primary prevention were required to have at least one cardiovascular risk factor, e.g., the Prospective Study of Pravastatin in the Elderly at Risk (PROSPER) trial $^{26)}$ or elevated C-reactive protein level, e.g., Justification for the Use of statins in Primary prevention: an Intervention Trial Evaluating Rosuvastatin (JUPITER) trial ${ }^{27)}$. In contrast, the present study included patients with hypercholesterolemia without cardiovascular diseases irrespective of risk factors.

Although the secondary prevention effect on statin treatment in old age is clearly beneficial, the effect on primary prevention has 

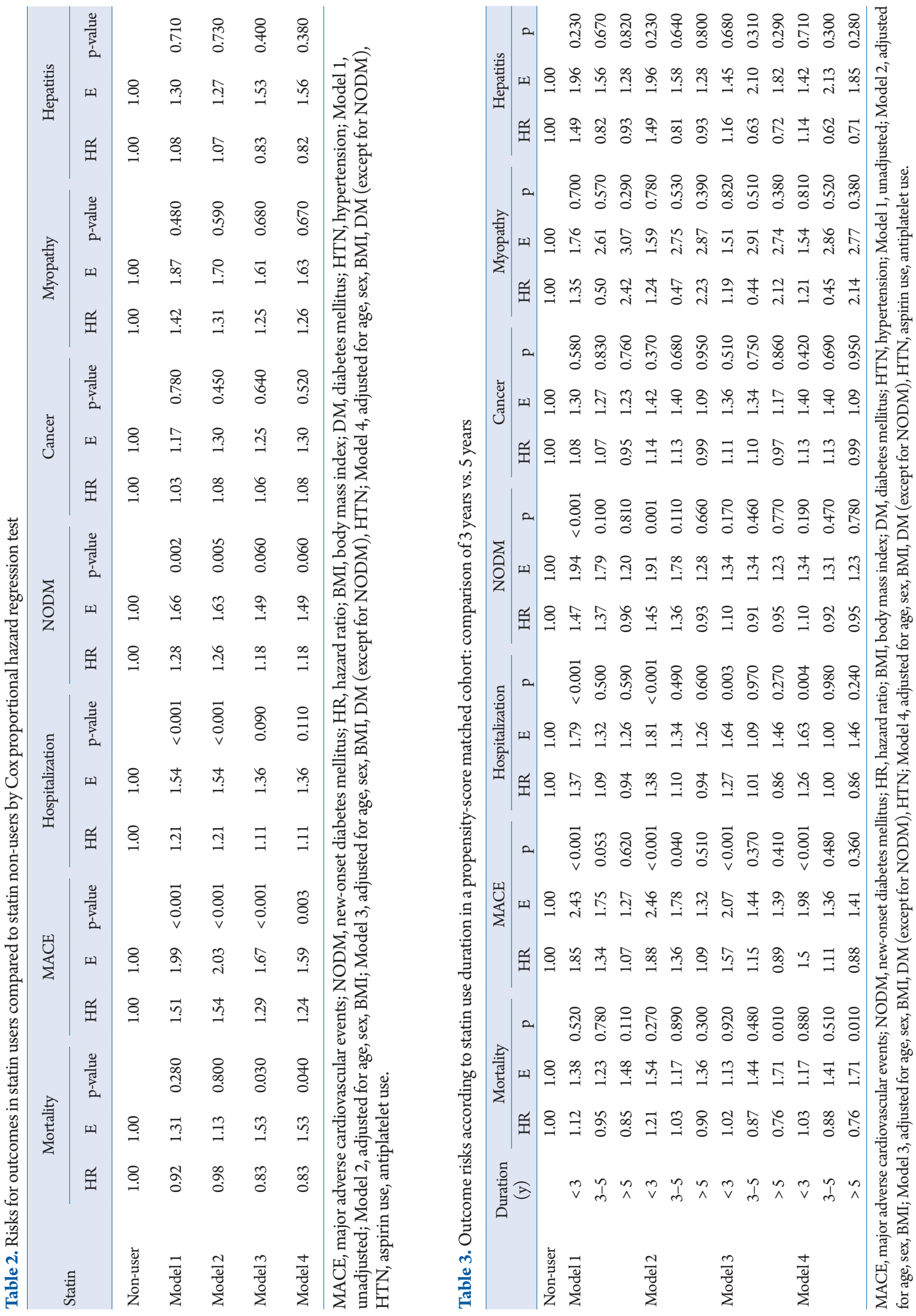
not been clear in randomized controlled trial studies. The PROSPER study was the first to investigate the effect of statin use in older adults without cardiovascular disease. ${ }^{26)}$ The results indicated a $15 \%$ decreased risk of cardiovascular disease in the pravastatin group than in the placebo group, but no reduction in all-cause mortality $(\mathrm{HR}=0.85 ; 95 \%$ confidence interval $[\mathrm{CI}], 0.77-1.15)$. In the JUPITER study, the use of rosuvastatin did not significantly reduce mortality ( $\mathrm{HR}=0.8 ; 95 \% \mathrm{CI}, 0.62-1.04)$ in those aged over 70 years without cardiovascular disease, with an LDL-C level $<130 \mathrm{mg} / \mathrm{dL}$ and high-sensitivity (hs)-CRP level of $2 \mathrm{mg} / \mathrm{L}$ or higher, but it did decrease cardiovascular disease incidence ${ }^{27)}(\mathrm{HR}=0.61 ; 95 \% \mathrm{CI}$, 0.46-0.82). In the Cholesterol Treatment Trialists, meta-analysis of subjects over 75 years of age did not prove the primary prevention effect $\left.^{4}\right)(\mathrm{HR}=0.92 ; 95 \% \mathrm{CI}, 0.73-1.16)$.

The results of observational studies have suggested mixed and less obvious benefits of initiating statins in patients older than 75 years with increased risks of cardiovascular disease, such as diabetics (e.g., Ramos et al. ${ }^{28)}$ ), with cardiovascular disease risk factors (e.g., the Statins on Clinical Outcomes for Primary prevention in individuals aged $>75$ years $\left(\right.$ SCOPE-75) trial $^{29)}$ ), or in men (e.g., Orkaby et al. ${ }^{30)}$ ).

In the present study, statin use for primary prevention in patients aged over 75 years increased the risk of MACE for the first 3 years. Physicians may have been more likely to prescribe statins to patients with veiled cardiovascular risk factors that were not registered on claim diagnostic codes, which could have increased the healthy user selection bias. ${ }^{31)}$

However, our sub-analyses of use duration showed that the risk of MACE, hospitalization, and NODM decreased after 5 years of starting statin prescription. This result is consistent with Mansi's finding that statin users had significantly higher odds of developing diabetes (odds ratio $[\mathrm{OR}]=1.93$; 95\% CI, 1.55-2.41), which persisted throughout follow-up and that short-term statin use was not associated with decreased odds of major acute cardiovascular events $\left(\mathrm{OR}=1.17\right.$; 95\% CI, 0.72-1.92). ${ }^{32)}$ These results indicate that statins may be effective for primary prevention when used long-term (e.g., 5 years or more).

Meanwhile, concern about statin use in older adults is related to fear of myalgia, increased fall risk, liver enzyme elevation, cognitive impairment, stroke, fatigue, and drug interactions in polypharmacy patients. ${ }^{33,34)}$ However, in this study, we observed no differences in the risks of myopathy and hepatitis between statin users and non-users. Moreover, statin therapy was not associated with elevated risks of cancer or hospitalization. Our results are similar to the those of the 2015 the Patient and provider Assessment of Lipid Management (PALM) registry, in which statins appeared to be similarly tolerated in older ( $>75$ years) and younger adults. ${ }^{35)}$
Our study has several limitations. First, there was the possibility of coding errors, missing data, lack of clinically relevant data because of unmeasured variables, or missing relevant drug use that was not typically collected in nationally-based datasets. Second, this was an observational study; hence, it suffers from potential selection bias despite robust exact block matching. One possible selection bias was that frail people might have been less likely to be prescribed statins. However, we observed no difference in physical activity between statin users and non-users and the prevalence of diabetes and HTN, which are potential risk factors for frailty, was higher in the statin group. These findings indicate that it was unlikely that the statin non-user group included more frail population. Moreover, patients with severe frailty were not likely to be included in this study because they could not have visited health examination centers and were excluded. Third, our study may be influenced by an immortal time bias. Statin users with MPR > 20\% during the observation period must survive to receive consecutive prescriptions and were, thus, less likely to die, which may have biased the increased mortality in the statin user group. However, our analysis of mortality as of the median time of survival duration showed no difference between statin users and non-users. Thus, we postulate the risk of immortal time bias might be less substantial. Fourth, we did not compare LDL-C, HDL cholesterol, and triglyceride levels between statin users and non-users, which could have affected the results, partly because LDL-C measurement was not included in the national health exam and triglyceride and HDL measurements were introduced in 2009. Fifth, we could not accurately confirm the cumulative or dose effects of statins because we could not determine the daily dose of statins from the available data. Sixth, while alcohol and smoking are risk factors for mortality, they did not differ significantly between statin users and non-users. Therefore, they were not confounding factors and were excluded from the adjustment. Seventh, there was a possibility of unknown confounding regarding adverse outcomes in the statistical analysis, despite the exact block matching method in consideration of this concern.

Despite these limitations, this nationwide study included a large sample of the older Korean adult population ( $>75$ years) and demonstrated the benefit of statins for primary prevention if used for more than 5 years in terms of reducing all-cause mortality. In conclusion, the results of our study suggested that the use of statins for primary prevention by adults aged over 75 years was associated with a significant lower mortality risk.

\section{ACKNOWLEDGMENTS}

\section{CONFLICT OF INTEREST}

The researchers claim no conflicts of interest. 


\section{FUNDING}

This study was supported grants by the Korea Geriatrics Society. The National Health Information Database was provided by the National Health Insurance Service of Korea.

\section{AUTHOR CONTRIBUTIONS}

Conceptualization, WCW, KSY; Data curation, CHS; Funding acquisition, KSY; Investigation, WCW, KSY; Methodology, WCW, KSY, CHS; Project administration, KSY, CHS; Supervision, WCW, KSY; Writing original draft, KSY; Writing-review \& editing, KSY, WCW.

\section{Supplementary Materials}

Supplement materials can be found via https://doi.org/10.4235/ agmr.20.0028.

\section{REFERENCES}

1. Jang IY, Lee HY, Lee E. Geriatrics fact sheet in Korea 2018 from national statistics. Ann Geriatr Med Res 2019;23:50-3.

2. Korean Statistical Information Service. Causes of death in 2018 [Internet]. Daejeon, Korea: Korean Statistical Information Service; c2020 [cited 2020 Jun 22]. Available from: http://kosis.kr/ statHtml/statHtml.do? orgId = 101\&tblId=DT_1B34E01\&conn_path $=\mathrm{I} 2$.

3. Veniant MM, Sullivan MA, Kim SK, Ambroziak P, Chu A, Wilson $\mathrm{MD}$, et al. Defining the atherogenicity of large and small lipoproteins containing apolipoprotein B100. J Clin Invest 2000;106:1501-10.

4. Cholesterol Treatment Trialists' (CTT) Collaboration. Efficacy and safety of more intensive lowering of LDL cholesterol: a meta-analysis of data from 170,000 participants in 26 randomised trials. Lancet 2010;376:1670-81.

5. Stone NJ, Robinson JG, Lichtenstein AH, Bairey Merz CN, Blum CB, Eckel RH, et al. 2013 ACC/AHA guideline on the treatment of blood cholesterol to reduce atherosclerotic cardiovascular risk in adults: a report of the American College of Cardiology/American Heart Association Task Force on Practice Guidelines. J Am Coll Cardiol 2014;63(25 Pt B):2889-934.

6. US Preventive Services Task Force. Statin use for the primary prevention of cardiovascular disease in adults: US Preventive Services Task Force Recommendation Statement. JAMA 2016;316:1997-2007.

7. Mortensen MB, Falk E. Primary prevention with statins in the elderly.J Am Coll Cardiol 2018;71:85-94.

8. Rabar S, Harker M, O’Flynn N, Wierzbicki AS; Guideline De- velopment Group. Lipid modification and cardiovascular risk assessment for the primary and secondary prevention of cardiovascular disease: summary of updated NICE guidance. BMJ 2014; 349:g4356.

9. Anderson TJ, Gregoire J, Pearson GJ, Barry AR, Couture P, Dawes M, et al. 2016 Canadian Cardiovascular Society Guidelines for the management of dyslipidemia for the prevention of cardiovascular disease in the adult. Can J Cardiol 2016;32:126382.

10. Piepoli MF, Hoes AW, Agewall S, Albus C, Brotons C, Catapano AL, et al. 2016 European Guidelines on cardiovascular disease prevention in clinical practice: the Sixth Joint Task Force of the European Society of Cardiology and Other Societies on Cardiovascular Disease Prevention in Clinical Practice (constituted by representatives of 10 societies and by invited experts) Developed with the special contribution of the European Association for Cardiovascular Prevention \& Rehabilitation (EACPR). Eur Heart J 2016;37:2315-81.

11. Grundy SM, Stone NJ, Bailey AL, Beam C, Birtcher KK, Blumenthal RS, et al. 2018 AHA/ACC/AACVPR/AAPA/ABC/ ACPM/ADA/AGS/APhA/ASPC/NLA/PCNA Guideline on the Management of Blood Cholesterol: Executive Summary: a report of the American College of Cardiology/American Heart Association Task Force on Clinical Practice Guidelines. J Am Coll Cardiol 2019; 73:3168-209.

12. Korean Academy of Medicine Science. Evidence-based guideline for dyslipidemia in primary care. Cheongju, Korea: Korean Academy of Medicine Science, Korea Centers for Disease Control and Prevention; 2018.

13. Gillmann K, El Ameen A, Massy R, Fabro F, Gasc A, Herbort CP Jr. Assessment of measurement methods of posterior inflammation in stromal choroiditis: the value of quantitative outcome measures versus the presently qualitatively based paradigm. Int Ophthalmol 2019;39:1567-74.

14. Ott BR, Daiello LA, Dahabreh IJ, Springate BA, Bixby K, Murali $\mathrm{M}$, et al. Do statins impair cognition? A systematic review and meta-analysis of randomized controlled trials. J Gen Intern Med 2015;30:348-58.

15. Kwon S. Thirty years of national health insurance in South Korea: lessons for achieving universal health care coverage. Health Policy Plan 2009;24:63-71.

16. Lee J, Lee JS, Park SH, Shin SA, Kim K. Cohort Profile: The National Health Insurance Service-National Sample Cohort (NHIS-NSC), South Korea. Int J Epidemiol 2017;46:e15.

17. Hwang D, Kim S, Choi H, Oh IH, Kim BS, Choi HR, et al. Calcium-channel blockers and dementia risk in older adults - National Health Insurance Service-Senior Cohort (2002-2013). Circ J 
2016;80:2336-42.

18. Garcia-Perez LE, Alvarez M, Dilla T, Gil-Guillen V, Orozco-Beltran $\mathrm{D}$. Adherence to therapies in patients with type 2 diabetes. Diabetes Ther 2013;4:175-94.

19. Deedwania P, Stone PH, Bairey Merz CN, Cosin-Aguilar J, Koylan N, Luo D, et al. Effects of intensive versus moderate lipid-lowering therapy on myocardial ischemia in older patients with coronary heart disease: results of the Study Assessing Goals in the Elderly (SAGE). Circulation 2007;115:700-7.

20. Ko SH, Kim DJ, Park JH, Park CY, Jung CH, Kwon HS, et al. Trends of antidiabetic drug use in adult type 2 diabetes in Korea in 2002-2013: Nationwide population-based cohort study. Medicine (Baltimore) 2016;95:e4018.

21. Cziraky MJ, Willey VJ, McKenney JM, Kamat SA, Fisher MD, Guyton JR, et al. Statin safety: an assessment using an administrative claims database. Am J Cardiol 2006;97:61C-68C.

22. Prospective Studies Collaboration. Blood cholesterol and vascular mortality by age, sex, and blood pressure: a meta-analysis of individual data from 61 prospective studies with 55,000 vascular deaths. Lancet 2007;370:1829-39.

23. Strandberg TE, Kolehmainen L, Vuorio A. Evaluation and treatment of older patients with hypercholesterolemia: a clinical review. JAMA 2014;312:1136-44.

24. Cholesterol Treatment Trialists' Collaboration. Efficacy and safety of statin therapy in older people: a meta-analysis of individual participant data from 28 randomised controlled trials. Lancet 2019;393:407-15.

25. Roth GA, Johnson C, Abajobir A, Abd-Allah F, Abera SF, Abyu $\mathrm{G}$, et al. Global, regional, and national burden of cardiovascular diseases for 10 causes, 1990 to 2015. J Am Coll Cardiol 2017;70:1-25.

26. Shepherd J, Blauw GJ, Murphy MB, Bollen EL, Buckley BM, Cobbe SM, et al. Pravastatin in elderly individuals at risk of vascular disease (PROSPER): a randomised controlled trial. Lancet 2002;360:1623-30.
27. Glynn RJ, Koenig W, Nordestgaard BG, Shepherd J, Ridker PM. Rosuvastatin for primary prevention in older persons with elevated C-reactive protein and low to average low-density lipoprotein cholesterol levels: exploratory analysis of a randomized trial. Ann Intern Med 2010;152:488-96.

28. Ramos R, Comas-Cufi M, Marti-Lluch R, Ballo E, Ponjoan A, Alves-Cabratosa L, et al. Statins for primary prevention of cardiovascular events and mortality in old and very old adults with and without type 2 diabetes: retrospective cohort study. BMJ 2018;362:k3359.

29. Kim K, Lee CJ, Shim CY, Kim JS, Kim BK, Park S, et al. Statin and clinical outcomes of primary prevention in individuals aged $>75$ years: The SCOPE-75 study. Atherosclerosis 2019;284:316.

30. Orkaby AR, Gaziano JM, Djousse L, Driver JA. Statins for primary prevention of cardiovascular events and mortality in older men. J Am Geriatr Soc 2017;65:2362-8.

31. Emilsson L, Garcia-Albeniz X, Logan RW, Caniglia EC, Kalager M, Hernan MA. Examining bias in studies of statin treatment and survival in patients with cancer. Version 2. JAMA Oncol 2018;4:63-70.

32. Mansi IA, English J, Zhang S, Mortensen EM, Halm EA. Longterm outcomes of short-term statin use in healthy adults: a retrospective cohort study. Drug Saf 2016;39:543-59.

33. Strandberg TE. Role of statin therapy in primary prevention of cardiovascular disease in elderly patients. Curr Atheroscler Rep 2019;21:28.

34. Odden MC, Pletcher MJ, Coxson PG, Thekkethala D, Guzman D, Heller D, et al. Cost-effectiveness and population impact of statins for primary prevention in adults aged 75 years or older in the United States. Ann Intern Med 2015;162:533-41.

35. Nanna MG, Navar AM, Wang TY, Mi X, Virani SS, Louie MJ, et al. Statin use and adverse effects among adults $>75$ years of age: insights from the patient and provider assessment of lipid management (PALM) registry.J Am Heart Assoc 2018; 7:e008546. 